\title{
Self-excited hydrothermal waves in evaporating sessile drops
}

\author{
K. Sefiane, ${ }^{1, a)}$ J. R. Moffat, ${ }^{1}$ O. K. Matar, ${ }^{2}$ and R. V. Craster $^{3}$ \\ ${ }^{1}$ School of Engineering and Electronics, The University of Edinburgh, Kings Buildings, Edinburgh EH9 \\ 3JL, United Kingdom \\ ${ }^{2}$ Department of Chemical Engineering, Imperial College London, London SW7 2AZ, United Kingdom \\ ${ }^{3}$ Department of Mathematics, Imperial College London, London SW7 2AZ, United Kingdom
}

(Received 11 June 2008; accepted 15 July 2008; published online 22 August 2008)

\begin{abstract}
Pattern formation driven by the spontaneous evaporation of sessile drops of methanol, ethanol, and FC-72 using infrared thermography is observed and, in certain cases, interpreted in terms of hydrothermal waves. Both methanol and ethanol drops exhibit thermal wave trains, whose wave number depends strongly on the liquid volatililty and substrate thermal conductivity. The FC-72 drops develop cellular structures whose size is proportional to the local thickness. Prior to this work, hydrothermal waves have been observed in the absence of evaporation in shallow liquid layers subjected to an imposed temperature gradient. In contrast, here both the temperature gradients and the drop thickness vary spatially and temporally and are a natural consequence of the evaporation process. (C) 2008 American Institute of Physics. [DOI: 10.1063/1.2969072]
\end{abstract}

We identify spontaneously occurring hydrothermal waves (HTWs) and patterns in evaporating droplets; HTWs are thermally induced traveling waves, which occur in the absence of surface deflection, and obtain their energy via the application of temperature gradients. ${ }^{1-3}$ Previous observations of HTWs have been reliant upon fixed prescribed temperature gradients and constant thickness fluid layers, and have not been observed during evaporation. Smith and Davis $^{2}$ predicted that fluid layers subjected to sufficiently large horizontal temperature gradients become unstable and exhibit steady longitudinal rolls or unsteady hydrothermal waves; the presence of these waves has been confirmed by several experimental investigations. ${ }^{4,5}$ Our interest is primarily in the latter scenario. HTWs have been observed primarily in thin fluid layers subjected to relatively small temperature gradients. They propagate parallel and almost perpendicularly to the temperature gradient for small and large Prandtl numbers, Pr, respectively. ${ }^{6}$ HTWs are unsteady and an inherently three-dimensional effect.

HTWs have also been observed in differentially heated pools of ethanol ${ }^{4}$ and silicon oil. ${ }^{5,6}$ There, HTWs resemble spirals at relatively large values of the thickness, and for films whose depth is less than the capillary length scale, the observed HTWs propagate radially from the inner to the outer edge of the cylinder. Temperature fluctuations associated with HTWs propagating in an annulus have also been reported; ${ }^{7}$ the amplitude and frequency of these oscillations depend on the imposed temperature gradient as well as the fluid depth. Evaporation, and its potential to spontaneously generate HTWs, has not previously been investigated. In this letter, we examine the case of a sessile drop undergoing evaporation and show how the temperature gradient across the drop, which arises naturally during the evaporation process, gives rise to instabilities involving HTWs.

The experimental setup consists of a syringe pump, a horizontal substrate mount, temperature controller, and infrared (IR) analysis equipment. Volatile sessile droplets evaporating on various solid substrates are investigated using IR thermography. We present results for four liquids: water, eth-

${ }^{\text {a)} E l e c t r o n i c ~ m a i l: ~ k . s e f i a n e @ e d . a c . u k . ~}$ anol, methanol, and FC-72. These are chosen to have a range of volatilities and their boiling points are 100, 78.3, 64.7, and $56{ }^{\circ} \mathrm{C}$, respectively, under atmospheric conditions. Additionally, we use four substrates made of PTFE, Macor (ceramic), titanium, and copper, having a range of thermal conductivities $0.25,1.46,21.9$, and $401 \mathrm{~W} \mathrm{~m}^{-1} \mathrm{~K}^{-1}$, respectively. Care was taken with substrates to ensure homogeneous wettabilities and accurate contact angle measurement; to this end, the substrates were coated with a very thin layer of a fluoropolymer Cytop.

To obtain images of the drop behavior, the IR camera was mounted directly above the substrate, facing vertically downwards onto the evaporating drop. The IR camera used was a FLIR ThermaCAM SC3000, which has a range of $-20-20000{ }^{\circ} \mathrm{C}$ and a thermal sensitivity of $20 \mathrm{mK}$. The images are recorded at 50 frames/s using a camera fitted with a microscope lens with a $10 \times 7.5 \mathrm{~mm}^{2}$ field of view and a minimum focal length of $26 \mathrm{~mm}$. The images acquired are transferred to a PC with built-in THERMACAM software. The spatial resolution of the system is $8-9 \mu \mathrm{m}$. In addition to IR thermography measurements, a drop shape analysis goniometer (Kruss DSA100) was used to measure the drop volume and the evaporation rate. The investigated drops have contact lines that remain pinned to the substrate for the majority of their lifetimes. Hence, the basal radius remained essentially constant while both the height and wetting angle decreased with time.

We recorded the interfacial thermal activity of the methanol, ethanol, and FC-72 drops. In the case of water drops, the thermal activity was comparatively weaker and the temperature remained approximately uniform spatially [see Fig. 1(a)]. This is supported by the recent work of Girard et al. ${ }^{8}$ who showed the magnitude of the temperature gradients that accompany the evaporation of water droplets to be small. The thermal patterns observed in the cases of methanol and ethanol, are similar to each other, resembling a wave train of alternating warm and warm/cool regions, which travel in the azimuthal direction (see supplementary material $^{9}$ for visualization). These patterns are characterized by the relatively dark, spokelike bands, as shown in Fig. 1(b) and these wave trains are reminiscent of HTWs observed in thin annular pools of silicon oil subjected to differential heat- 

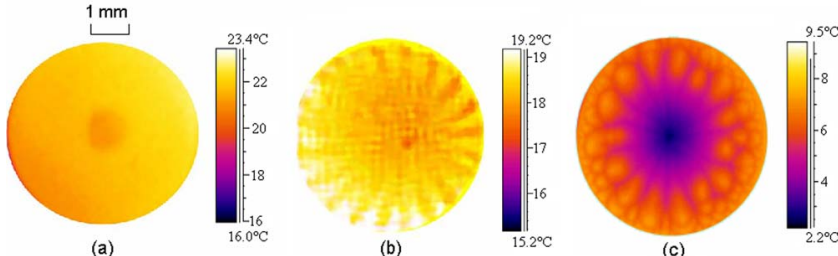

(b)

FIG. 1. (Color online) Patterns in sessile drops of water, methanol, and FC-72 refrigerant evaporating on silicon substrates, shown in panels (a)-(c), respectively, obtained using IR thermography. Movies of the process shown for methanol and FC-72 are available online (Ref. 9).

ing. No preferred direction of propagation of these waves was observed: both clockwise and anticlockwise propagation was recorded; in certain cases, the waves appeared to emanate from a source on the drop surface. The angle of propagation, its definition and its dependence on the relevant system parameters, are discussed below.

It is interesting to contrast the patterns arising for the different liquids. In FC-72 drops, thermally driven cells rather than HTWs were observed emerging near the drop center and drifting toward the edge. The cells were larger near the drop apex than in the vicinity of the contact line [see Fig. 1(c)]. In all cases considered, no interfacial waviness was discernible. We shall focus on the results generated for methanol and ethanol and then describe those associated with FC-72.

Waves on the surface of the methanol and ethanol drops, corresponding to the number of warmer (or cooler) spokelike regions, were visually counted and reported as a function of the dimensionless time $t / t_{\text {tot }}$, as shown in Fig. 2, where $t_{\text {tot }}$ represents the duration of an experimental run. Here, the error bars signify inaccuracies in the visual observation of the number of waves present. Inspection of this figure reveals that the number of waves depends on the thickness of the drop: as the evaporative process proceeds, the height of the drop decreases as does the number of visible waves, approximately linearly with time. The number of waves associated with the evaporating methanol drop is larger than that of the ethanol drop. The wavelike unsteady motion of these waves is clearly observed in the supplementary video material and is completely unlike the behavior of rolls. ${ }^{9}$

The effect of substrate thermal conductivity on the characteristics of the wave trains was also investigated. In Fig. 3, we show the dependence of the total number of observed

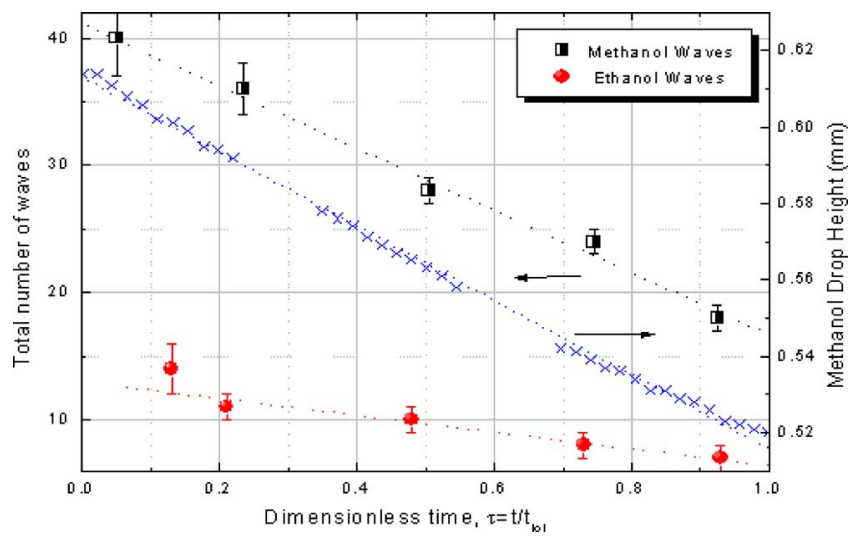

FIG. 2. (Color online) Temporal evolution of the number of observed waves for methanol and ethanol drops evaporating on a titanium substrate; the drop height evolution of a methanol drop is also shown. Here, $t_{\text {tot }}$ represents the time interval during which the experimental run was performed.

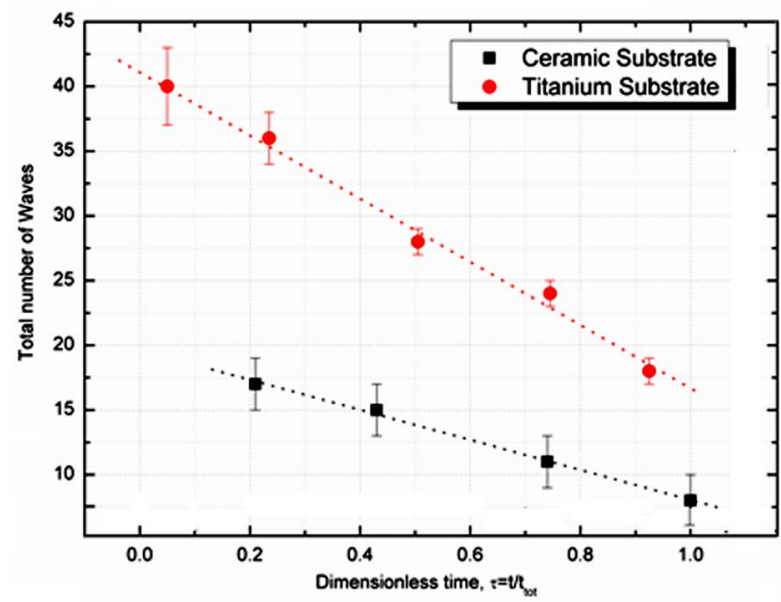

FIG. 3. (Color online) Temporal variation of the number of observed waves for methanol drops on ceramic and titanium substrates.

waves against $t / t_{\text {tot }}$ recorded for two substrates: Macor (ceramic) and titanium. For the sake of brevity, only the results for the most volatile liquid used, methanol, are presented. In addition to the previously observed trend of decreasing wave number with time, the number of waves associated with the higher conductivity substrate, titanium, is substantially larger than that associated with that of the less thermally conductive ceramic substrate. The reproducibility of the data was verified by conducting several runs for each liquid/substrate combination, and independently analyzing the results.

We turn our attention to the patterns observed during the evaporation of the FC-72 drops. Figure 4 shows the evolution of the patterns at various stages of the lifetime of the drop evaporating over a copper substrate. It can be seen clearly in Fig. 4(a) that, at early times, the drop develops a relatively cool region near its apex. The average interfacial temperature increases radially and exhibits a maximum near the drop edge.

During the course of the evaporation process, the minimal temperature at the apex and the extent of the cool region are reduced and the overall temperature gradient between the apex and the drop edge decreases [see Figs. 4(b) and 4(c)]. It is also seen in Fig. 4 that the cells, which are evident at early times, appear to extend from the flow origin to region immediately upstream of the contact line [see Fig. 4(a)]; in the region nearest the contact line, cells of smaller dimension are discernible. With increasing time and decreasing drop height, the extent of the region populated by the larger cells decreases and appears to become increasingly confined to the center of the drop; the region occupied by the smaller cells, on the other hand, increases substantially. It is important to note that, in contrast to the ethanol and methanol drops, the patterns observed in the case of FC-72 drops do not correspond to propagating waves.
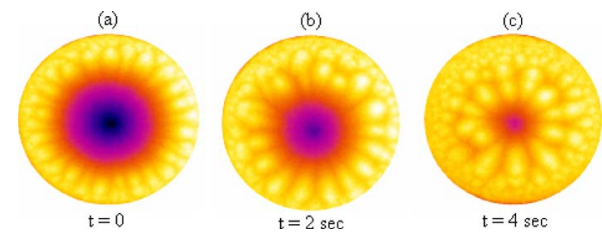

FIG. 4. (Color online) Evolution of the patterns observed during the evaporation of a FC-72 drop on a titanium substrate. The time interval separating consecutive snapshots in (a)-(c) is $2 \mathrm{~s}$. 
TABLE I. Estimates of relevant dimensionless parameters.

\begin{tabular}{lccc}
\hline \hline & Methanol & Ethanol & FC-72 \\
\hline $\mathrm{Ma}=\gamma H^{2} \Delta T / \rho \nu \kappa l$ & $8.7 \times 10^{2}$ & $3.6 \times 10^{3}$ & $4.9 \times 10^{2}$ \\
$\mathrm{Ra}=\alpha g H^{4} \Delta T / \nu \kappa l$ & 58.6 & 16.8 & 10.2 \\
$\chi=\mathrm{Ra} / \mathrm{Ma}$ & $6.7 \times 10^{-2}$ & $4.7 \times 10^{-3}$ & $2.1 \times 10^{-2}$ \\
$\mathrm{Bo}=\rho g H^{2} / \sigma$ & 0.16 & 0.13 & 0.11 \\
\hline \hline
\end{tabular}

Temperature fluctuations in the azimuthal direction associated with the wave trains in methanol were measured (not shown) by recording the temporal variation of the temperature at a chosen point at the drop periphery; fluctuations are in the range $\pm 0.5 \mathrm{~K}$. Since the formation of wave trains is absent in the case of FC-72, temperature fluctuations were obtained by direct observation of the IR images rather than by the method used for methanol. These fluctuations were found to be in the range of $1-2 \mathrm{~K}$ and, therefore, more pronounced than in the methanol case.

We rationalize and interpret our experiments using recent work by Garnier et al. (see Ref. 10, and references therein) who studied the thermocapillary-driven patterns in shallow annular pools of silicon. They observe the formation of HTWs when the inner and outer walls of the annular container have different imposed temperatures and thus generate a temperature gradient across the layer. They report the development of HTWs particularly in situations wherein the outer wall is hotter than the inner one. Garnier et al. ${ }^{10}$ correlate their results by estimating the values of the Marangoni, Rayleigh, and Bond numbers, given by $\mathrm{Ma}$, Ra, and Bo, defined in Table I. Here, $T$ denotes the temperature, $H$ and $l$ are the characteristic vertical and radial length scales, $\rho, \nu$, and $\kappa$ are the density, kinematic viscosity, and thermal diffusivity, respectively; $g$ is the gravitational acceleration, $\sigma$ is the surface tension; $\alpha=(1 / \rho)(\partial \rho / \partial T)$ is the coefficient of thermal expansion and $\gamma=-(\partial \sigma / \partial T)$, a positive quantity for most pure fluids. It is also useful to define $\chi$ : for $\chi \ll 1$ one would expect to observe thermocapillary-driven waves and then beyond a threshold to observe rolls.

For the liquids used here, estimates for the dimensionless parameters are listed in Table I; these were obtained using average values for $\Delta T, H$, and $l$. Notably $\chi \ll 1$ for both methanol and ethanol which indicates that thermocapillary forces dominate those related to buoyancy. Thus, for these liquids, the dynamics should be accompanied by the formation of thermocapillary-driven patterns such as the HTWs observed by Garnier et al. ${ }^{10}$ This is entirely consistent with the observations of thermally induced waves accompanying the evaporation of methanol and ethanol drops. From Table I, gravitational effects are expected to play a relatively minor role since Bo is much less than unity, indicating surface tension dominance. From Table I, it is seen that the value of $\chi$ indicates that thermocapillary forces should be dominant in the case of FC-72. This, however, is not borne out by the patterns shown in Figs. 1 and 4 above, which resemble rolls rather than hydrothermal waves.

The findings shown in Fig. 3 are consistent with a HTWdriving mechanism: as shown by Girard et al. ${ }^{8}$ the magnitude of the temperature gradients that accompany the evaporation of a sessile drop decreases with increasing time and decreasing drop thickness. This leads to a concomitant decrease in the driving force for the formation of traveling wave trains.
The results shown in Fig. 4 are also explained by recalling that the energy lost due to evaporation, which is maximal at the contact line, can be compensated provided the ratio of substrate to liquid thermal conductivity is sufficiently large. ${ }^{11-13}$

It is important to note that in contrast with Ref. 10 as well as in other cases in which thermally induced waves result from the imposition of constant temperature gradients, these gradients in the case of evaporating sessile drops are self-generated due to the spatially nonuniform evaporative flux. Furthermore, the conditions of criticality are now both spatially and temporally varying due to the evolving drop height and temperature gradients; these features are absent in the case of differentially heated pools.

The angle of propagation of the thermally induced wave trains, which measures the deviation of the axis of an individual wave from the radial direction, was also measured for methanol and ethanol. Previous work has shown this quantity to be strongly dependent on the Prandtl number, $\mathrm{Pr}$ $=\mathrm{Cp} \mu / \lambda$, for HTWs ${ }^{2}$ here, $\mathrm{Cp}, \mu$, and $\lambda$ denote the specific heat capacity, dynamic viscosity, and thermal conductivity, respectively. Our results indicate that the propagation angles for methanol and ethanol ( $\mathrm{Pr}=6.4$ and 12 , respectively) are $11^{\circ}$ and $20^{\circ}$ for PTFE, $14^{\circ}$ and $25^{\circ}$ for PTFE, and $19^{\circ}$ and $28^{\circ}$ for PTFE, respectively. This finding is in line with the results of previous work on differentially heated thin liquid pools, which showed that the propagation angle increases with Pr. $^{2}$ Moreover, it appears that substrates of larger thermal conductivity, which lead to larger evaporation rates, also give rise to higher propagation angle.

In summary, we have studied the spontaneous evaporation of sessile drops of varying volatility on substrates with a wide range of thermal conductivity using IR thermography; attention was focused on the thermally induced dynamics that accompanies the evaporative process. We have presented experimental evidence for the presence of interfacial temperature wave trains, which, in the case of methanol and ethanol, are identified with the HTWs observed previously in the literature ${ }^{1-7}$ the dependence of the wave-train characteristics on system parameters provides strong supporting evidence allowing us to identify these waves as HTWs. It is important to note that, whereas in previous studies ${ }^{2,3,6,7}$ HTWs resulted from the imposition of a temperature gradient, the driving force for the wave trains in the volatile sessile droplets reported here, are now self-generated temperature gradients due to nonuniform evaporation. ${ }^{11}$

${ }^{1}$ S. H. Davis, Annu. Rev. Fluid Mech. 19, 403 (1987).

${ }^{2}$ M. K. Smith and S. H. Davis, J. Fluid Mech. 132, 119 (1983).

${ }^{3}$ M. K. Smith, Phys. Fluids 29, 3182 (1986).

${ }^{4}$ D. Schwabe, U. Möller, J. Schneider, and A. Scharmann, Phys. Fluids A 4, 2368 (1992).

${ }^{5}$ R. J. Riley and G. P. Neitzel, J. Fluid Mech. 359, 143 (1998)

${ }^{6}$ N. Garnier and A. Chiffaudel, Eur. Phys. J. B 19, 87 (2001).

${ }^{7}$ D. Schwabe, A. Zebib, and B. C. Sim, J. Fluid Mech. 491, 239 (2003).

${ }^{8}$ F. Girard, M. Antoni, S. Faure, and A. Steinchen, Langmuir 22, 11085 (2006)

${ }^{9}$ Movies of the process shown in Refs. 1 and 4 are available online http:// www.see.ed.ac.uk/ ksefiane/IR-waves/.

${ }^{10}$ N. Garnier, A. Chiffaudel, and F. Daviaud, Springer Tracts Mod. Phys. 207, 147 (2006).

${ }^{11}$ W. D. Ristenpart, P. G. Kim, C. Domingues, J. Wan, and H. A. Stone, Phys. Rev. Lett. 99, 234502 (2007).

${ }^{12}$ H. Mancini and D. Maza, Europhys. Lett. 66, 812 (2004).

${ }^{13}$ S. David, K. Sefiane, and L. Tadrist, Colloids Surf., A 298, 108 (2007). 